Journal of Applied Fluid Mechanics, Vol. 14, No. 6, pp. 1679-1689, 2021. Available online at www.jafmonline.net, ISSN 1735-3572, EISSN 1735-3645. https://doi.org/10.47176/jafm.14.06.32439

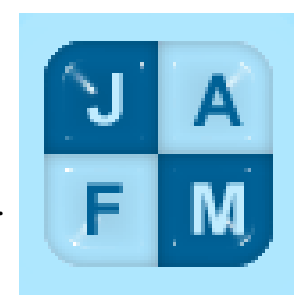

\title{
Aeroelastic Simulation of Stall Flutter Undergoing High and Low Amplitude Limit Cycle Oscillations
}

\author{
M. K. H. M. Zorkipli ${ }^{1}$, A. Abbas ${ }^{2}$ and N. A. Razak $^{1 \dagger}$ \\ ${ }^{1}$ School of Aerospace Engineering, Engineering Campus, University Sains Malaysia, Nibong Tebal, Penang, \\ 14300, Malaysia \\ ${ }^{2}$ School of Mechanical Engineering, Engineering Campus, University Sains Malaysia, Nibong \\ Tebal, Penang, 14300, Malaysia
}

†Corresponding Author Email: norizham@usm.my

(Received December 3, 2020; accepted May 10, 2021)

\begin{abstract}
The aeroelastic behaviour of an airfoil oscillating in large and small pitch amplitudes due to nonlinearity in aerodynamics is examined. The phenomenon of stall flutter resulted in the limit cycle oscillations of NACA 0012 at low to intermediate Reynolds number is investigated numerically through the unsteady twodimensional aeroelastic simulation. The simulations employed unsteady Reynolds Average Navier Stokes shear stress transport k- $\omega$ turbulent model with the low Reynolds number correction. The simulations of the fluidstructure interaction were performed by coupling the structural equation of motion with a fluid solver through the user-defined function utility. Numerical simulations were executed at three different elastic axis positions; the leading-edge, $18 \%$ and $36 \%$ of the airfoil chord length. The airfoil chord measures $0.156 \mathrm{~m}$. The simulations were executed at the free stream velocity ranging from $5.0 \mathrm{~m} / \mathrm{s}$ to $13 \mathrm{~m} / \mathrm{s}$ corresponding to the Reynolds number between 51618 and 134207. Two types of oscillation amplitudes were observed at each elastic axis position. At the leading-edge and $18 \%$ case, small amplitude oscillations were observed while at $36 \%$, the system underwent high amplitude oscillations. The analysis revealed the cause for small oscillation amplitude is due to the separation of the laminar boundary layer on the suction side of the airfoil starting at the trailing edge. High amplitude oscillations occurred due to the existence of the dynamic stall phenomenon beginning at the leading-edge. Small amplitude LCOs only occurred within a limited range of airspeed before it disappeared due to increasing airspeed.
\end{abstract}

Keywords: Stall flutter; Limit cycle oscillation; Flow separation.

\section{NOMENCLATURE}

$\begin{array}{llll}C_{\mathrm{p}} & \text { specific heat at constant pressure } & t & \text { time } \\ D_{h} & \text { inlet hydraulic diameter } & T_{i} & \text { initial temperature } \\ d & \text { thickness of plate } & T_{i n} & \text { temperature of inlet } \\ e & \text { Height of ribs } & T_{m} & \text { temperature of fluid } \\ f & \text { friction factor } & T_{w} & \text { temperature of wall } \\ f_{0} & \text { friction factor in smooth channel } & T P F & \text { Thermal Performance Factor } \\ H & \text { height of pin fin } & W & \text { width of channel } \\ h & \text { heat transfer coefficient } & x & \text { x direction of air flow } \\ k & \text { turbulence kinetic energy } & y & \text { y direction of air flow } \\ k l & \text { kinetic energy of laminar } & z & \text { z direction of air flow } \\ L & \text { streamwise length of the channel } & \alpha & \text { Thermal diffusivity } \\ N u & \text { Nusselt number } & \lambda & \text { fluid thermal conductivity } \\ N u_{0} & \text { Nusselt number in smooth channel } & \omega & \text { specific rate of dissipation } \\ R e & \text { Reynolds number } & v & \text { kinematic viscosity coefficient } \\ \mathrm{RNG} & \text { Re- Normalization Group } & \rho & \text { fluid density } \\ \mathrm{SST} & \text { Shear Stress Transfer } & \Delta P & \text { pressure drop between inlet and outlet } \\ T & \text { the temperature } & & \end{array}$




\section{INTRODUCTION}

Dynamic aeroelastic phenomena are phenomena resulting from the mutual interaction of aerodynamic forces, inertia forces and elastic forces on flexible structures. Limit cycle oscillation or LCOs is one of the vibration phenomena which requires at least one nonlinear element in a given aeroelastic system to exist (Razak and Dimitriadis 2013). In the aeroelastic system, the source of nonlinearity can either originate from structural, aerodynamic or both. Stall flutter is one of the phenomena that arise from nonlinear aeroelastic effects. Stall flutter is a phenomenon that occurs when the flow separates and re-attaches from any aerodynamic surface in a repeating manner (Raggett 1973). Complete or partial separation of flow over the suction side of the wing surface has been observed during stall flutter (Ericsson and Reding 1980).

Extensive works regarding stall flutter and oscillating wings have been performed to improve the understanding of the phenomenon. This work was later extended to include other wings profiles and a wider range of parameters. Wind tunnel experiments showed that the unsteady kinematic parameters play a crucial role in determining the unsteady aerodynamic loads generated during stall flutter (McCroskey et al. 1981; McCroskey 1981). Structural freeplay stall flutter was examined by Dimitriadis and Li (2009). They observed symmetrical and unsymmetrical bifurcation partly contributed by the dynamic stall phenomenon. Razak et al. (2011) investigated the effect of airspeed and the initial angle of attack on stall flutter behaviour. Their results revealed two levels of LCO amplitudes correspond to partial or complete separation around the wing. Partial separation manifest from the trailing edge, while large-amplitude LCOs were caused by separation originates from the leadingedge.

In this paper, the problem of fluid-structure interaction of self-excited pitching oscillation due to flow separation or stall flutter is investigated numerically. The purpose of the present work is to carry out numerical simulations on NACA 0012 that undergoes stall flutter at low to moderate Reynolds number. By varying the elastic axis positions, it is intended to evaluate the system's dynamics and aerodynamics characteristics that resulted in the selfsustain oscillations. The interaction between the fluid and elastic structure was achieved through coupling the unsteady aerodynamic forces and airfoil pitching motion together. The fluid side of the simulation was solved using the Navier Stokes equation with RANS approach. The structural side of the problem was solved using Newmark Beta time integration technique.

The evaluation of the effect of the elastic axis position on the dynamics and aerodynamics behaviour of an airfoil undergoing limit cycle oscillation due to flow separation and reattachment revealed some interesting findings. Different elastic axis position yield different dynamics and aerodynamics behaviour of the system. The difference in amplitude, frequencies and LCO onset airspeeds are highly dependent on the type and size of the unsteady flow separation and reattachment experience by the airfoil. As the elastic axis is shifted forward, the frequencies of the LCOs and LCO onset airspeeds increased as the LCO amplitudes decreased.

\section{Methodology}

\subsection{Computational Scheme}

The transient numerical simulations were conducted using ANSYS FLUENT 16.1. The Navier Stokes fluid solver is coupled with the elastically mounted rigid NACA 0012 airfoil. Spatial and temporal discretization is performed with second-order schemes for all quantities. The velocity pressure coupling is based on the PISO segregated algorithm for improved accuracy. The two-dimensional RANS shear stress transport (SST) $\mathrm{k}-\omega$ is used in this work to capture more robust additional flows around the airfoil surface (Ahmad et al. 2005). The calculations of the incompressible Reynolds Averaged NavierStokes (RANS) equation under Boussinesq's assumption is solved in the inertial frame of reference which requires relatively low computational cost when compared with higher fidelity methods. Boussinesq's assumption can accommodate many technical flows, such as flow near or around a wall boundary layer. It has been reported that this model is effective for low Reynolds number cases, provided that the mesh resolution is fine with a wall Y+ less than 1 (Aftab et al. 2016). The Navier-Stokes governing equations for twodimensional incompressible flow is given in Eqs. (1) and (2) where $U$ is the mean (ensemble-averaged) velocity vector, $\rho$ is the fluid density and $v_{t}$ is the turbulent kinematic eddy viscosity.

$$
\begin{aligned}
& \nabla . U=0 \\
& \frac{\partial u}{\partial t}+U . \nabla U=-\frac{1}{\rho} \nabla \rho+\left(v+v_{t}\right) \nabla^{2} U
\end{aligned}
$$

The time step size used for the RANS simulations is $\mathrm{dt}=0.00001 \mathrm{~s}$ and the iteration was set to 1000 per time step. It was found that the time step size is dependent on the mesh size for stable calculation. At each time step, iterations were carried out until a root mean square (RMS) convergence criterion of $10^{-4}$ on all residuals were reached. The simulation time was set to 4 seconds. There were cases where the trend of the oscillation amplitude, its maximum values, and oscillation frequency cannot be determined within 4 seconds. In such cases, the simulation time was extended until the trend can be determined. The run time for one complete simulation took two to three weeks to finish depending on the simulation time set. This was largely due to the small-time step value selected and the limitation in the computing power.

\subsection{Mesh and Boundary Conditions}

Figure 1(a) shows the computational domain for the stall flutter simulations. The fluid domain is separated into two regions, static and rotating region. 


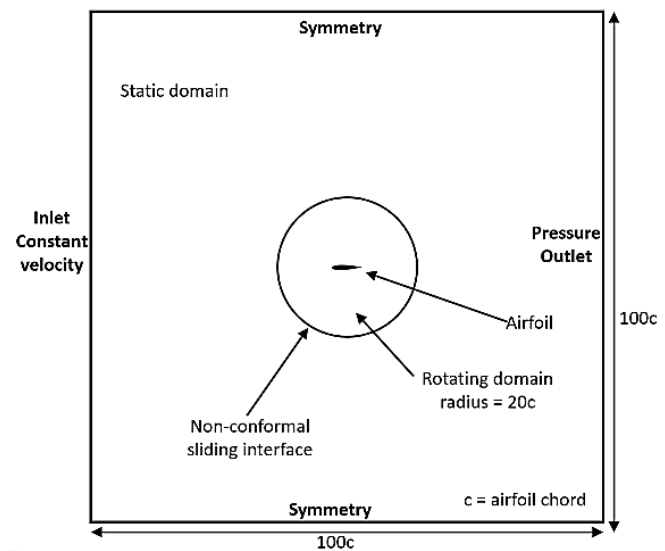

(a)

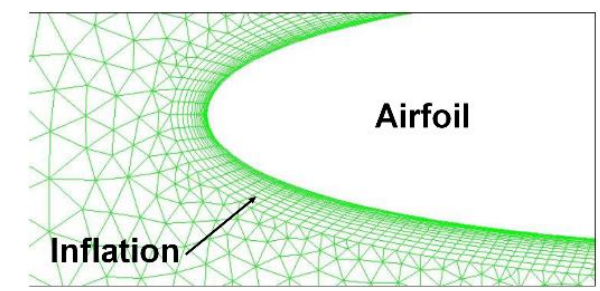

(b)

Fig. 1. (a) Fluid domain and

boundary conditions, (b) Airfoil mesh.

The radius of the rotating region was set at 20c, where $c$ is the airfoil chord length. The radius is measured from the position of the airfoil elastic axis. The static domain was set to $100 \mathrm{c}$. This value is chosen in order to avoid reversed flow error and provide enough distance for the wake and vortices to dissipate away from the airfoil (ANSYS, 2015). Reversed flow error can manifest if the computational fluid domain is too small for the fluid to flow. Constant and uniform velocity is imposed at the inlet while constant static pressure is imposed at the outlet. The upper and lower static domain is set as a symmetry boundary condition and the interior region is set as the fluid domain.

The combination of unstructured meshes is used in the flow domain along with the inflation structured meshes applied at the airfoil boundary layer as shown in Fig 1(b). The two-dimensional pitchoscillating airfoil is solved in the frame of reference which does not require mesh deformation and remeshing at the boundary layer in order to obtain better accuracy and speed up the calculations. A circular, non-conformal sliding interface which its centre located at the elastic axis is used. This allows only the inner part to rotate rigidly along with the airfoil. The outer part or static domain remains stationary throughout the simulation.

The periodic boundary condition is applied at the interfaces which allow the flow to enter and leave the rotating domain. The application circular nonconformal sliding interfaces and periodic boundary condition has been successfully employed by Kinsey and Dumas (2008) in their study of oscillating airfoil power extraction.

\subsection{Aeroelastic Model}

In this work, a one-degree of freedom pitch mounted rigid body airfoil is modelled based on the stall flutter aeroelastic system. The governing equation of motion for the system under consideration is shown in Eq. (3).

$$
I_{E A} \stackrel{\Pi}{\theta}+\stackrel{\sqcap}{D} \theta+K \theta=M_{E A}
$$

The expression $\theta$ denotes the pitch angle. The three parameters, $I_{E A}, D$, and $K$ are the vibration parameters. $M_{E A}$ on the right-hand side of Eq. (3) represents the aerodynamic moment evaluated at the elastic axis which is located at the positions of interest. IEA is the mass moment of inertia at the elastic axis. The other two parameters D and $\mathrm{K}$ are the viscous damping and structural stiffness respectively. Equation (3) is solved using the Newmark Beta direct time integration method. The Newmark Beta method is a method used in numerical evaluation of the dynamic response of structures. The Newmark Beta method is solved at every time step to provide pitch angle, pitch rate, and pitch acceleration at every time step. The Newmark Beta method is based on the following Eq. (4) and Eq. (5).

$\stackrel{\square}{\theta}_{t+d t}=\stackrel{\square}{\theta}_{t}+[(1-\gamma) d t] \stackrel{\mathbb{B}}{\theta}_{t}+(\gamma d t) \stackrel{\mathbb{\theta}}{\theta_{t+d t}}$

$\theta_{t+d t}=$

$\theta_{t}+d t \theta_{t}+\left[(0.5-\beta)(d t)^{2}\right]^{\mathbb{W}} \theta_{t}+\left[\beta(d t)^{2}\right] \theta_{t+d t}^{\mathbb{W}}$

The parameter $\beta$ and $\gamma$ define the variation of acceleration over a time step and determine the stability and accuracy characteristic of this integration method. The value for Newmark constants of $\beta$ and $\gamma$ is 0.25 and 0.5 respectively. These values are based on the average acceleration in the Newmark method (Newmark 1959; Chopra 1996).

The coupling of the structural and fluid equations is applied at each time step. At each time step, the pitching moment value is evaluated at the elastic axis position obtained from the fluid solver. This value is passed onto the structural solver. The structural solver is then executed to obtain the dynamic response of the airfoil in pitch. This process is repeated until convergence was achieved before the computation is marched forward in time. The converged updated position of the airfoil is then transferred back to the fluid solver which updates the mesh and airfoil position again before proceeding with the new fluid computation.

In Fluent, it is possible to solve the structural dynamic side using the Newmark Beta technique using the user-defined functions (UDF) utility to couple the structural solver with the fluid solver (Gang et al. 2019). At each time step, the fluid solver calculates the unsteady aerodynamics forces around the airfoil. Fluent fluid solver requires the angular pitch velocity provided from the UDF in order to 
update the mesh (Canonsburg 2015). The calculation steps are illustrated in Fig. 2.

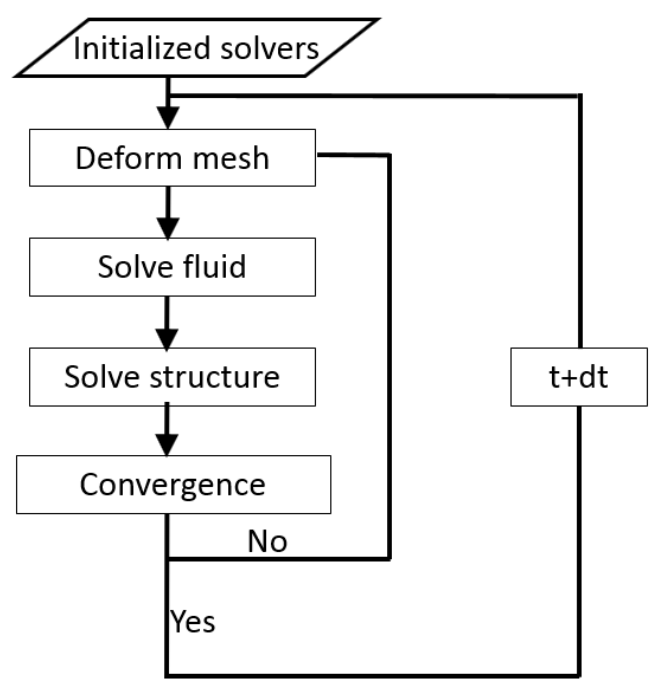

Fig. 2. FSI computation steps.

\subsection{Turbulence Modeling}

In this study, the RANS shear-Stress Transport (SST) $\mathrm{k}-\omega$ model is used. This model was developed by Menter to effectively blend the robust and accurate formulation of the standard $\mathrm{k}-\omega$ model (Menter 1994). It is the combination of a k- $\omega$ model and $\mathrm{k}-\varepsilon$ model which produce SST $\mathrm{k}-\omega$ which is reliable for a wider class of flows and provides a more accurate prediction of flow separation than other RANS models (ANSYS 2010). The LowReynolds correction option was employed in this simulation work. This option specifies a lowReynolds-number correction to the turbulent viscosity and allows for a more accurate representation of the actual flow physics. SST k- $\omega$ turbulent model is widely used for cases where a highly accurate resolution of the boundary layer is critical when involving flow separation at the boundary layer. The first distance from the boundary layer was set based on the Y plus value equal to one which is suitable for resolving the Viscous Sublayer in (SST) $\mathrm{k}-\omega$ model (Aftab et al. 2016). In this simulation, the turbulence intensity is set at $5 \%$ and the turbulent viscosity ratio is equal to 6 . These values are reasonable values for the simulations. Turbulent viscosity ratio range between 1 to 10 is typically good for external flows.

\subsection{Simulation Parameters}

The simulation parameters such as the air density and viscosity are considered at sea level conditions at $20^{\circ}$ C. The mass moment of inertia values about the elastic axis located at the leading-edge, $18 \%$ and $36 \%$ of the chord length measured from the leadingedge were maintained at $\mathrm{I}_{\theta}=0.00135 \mathrm{kgm}^{2}$. The linear structural stiffness value and viscous damping was $0.3 \mathrm{Nm} / \mathrm{rad}$ and $0.002 \mathrm{Nms} / \mathrm{rad}$ respectively. NACA 0012 airfoil was used as the test case airfoil. The airfoil has a chord length of 0.156 meters. The airspeed was varied corresponding to the freestream velocity ranges from $5 \mathrm{~m} / \mathrm{s}$ to $13 \mathrm{~m} / \mathrm{s}$ equivalent to Reynolds number of 51618 to 134207 . The simulation parameters are provided in Table 1 . In this simulation, the airfoil was given an initial condition in term of initial displacement where the pitch angle was set $0.2^{\circ}$ at the beginning of the simulation. Initial velocity was set to 0 degree/s. Before the simulation is marched in time, the airflow around the airfoil is initialized to the simulated airspeed before the airfoil is released from its initial condition.

It should be noted that the simulated airspeed range between the three cases mentioned is varied. The objective of the simulation is to observe selfsustained oscillation of stall flutter with the interest in finding the range of airspeed where the selfsustained oscillation occurs, i.e.; when it's appeared and disappeared. Since the stall flutter phenomenon cannot be predicted reliably using linear aeroelastic prediction methods, the first simulated airspeed was randomly chosen for the three cases. The first chosen airspeed provided a baseline where other simulation airspeeds were selected. The future airspeed selection depended upon whether the first simulated airspeed resulted in damped oscillation or selfsustained oscillation. If self-sustained oscillation was achieved, the future simulation airspeeds should be higher and lower than the first chosen airspeed. The increment and decrement of the airspeed were continued until decay responses were achieved in both directions.

Table 1 Simulation parameters.

\begin{tabular}{|l|l|}
\hline Variables & Value \\
\hline Pitch stiffness, K & $0.3 \mathrm{Nm} / \mathrm{rad}$ \\
\hline Pitch damping, D & $0.002 \mathrm{Nms} / \mathrm{rad}$ \\
\hline Mass moment of inertia, IEA & $0.00135 \mathrm{Kgm}^{2}$ \\
\hline Elastic axis (EA) in \% of chord & 1,18 and 36 \\
\hline Airfoil chord length (c) & $0.156 \mathrm{~m}$ \\
\hline
\end{tabular}

The alternate method would be to start the simulation at the lowest airspeed deem suitable (ideally $0 \mathrm{~m} / \mathrm{s}$ ) and increase the airspeed steadily until after selfsustained oscillation disappeared. This approach would take a considerable amount of simulation time which is not efficient and avoided. The reason different cases resulted in different airspeed ranges is theorized such that by changing the elastic axis, the dynamics and aerodynamics behaviour of the system is altered which resulted in different dynamic stability being exhibited.

\subsection{Mesh Convergence and Validation}

The numerical solutions are considered gridindependent if the truncation error is small enough and the simulation results change very little along with refining of the mesh. The purpose of the grid independence test is to determine the minimum 
number of nodes or elements required for a mesh to obtain good numerical results that are independent of the mesh resolution (Abu Bakar et al. 2019). The grid independence test involves refining the mesh elements and comparing solutions obtained from the coarse and fine meshes. In this work, five separated meshes with an increasing number of mesh elements from 50,000 to 250,000 are studied. All the test cases for mesh convergence were simulated with the airfoil pitching around the mean angle of attack of $2^{\circ}$ with $2^{\circ}$ pitch amplitude at an airspeed of $10 \mathrm{~m} / \mathrm{s}$. The pitch frequency was set to $5 \mathrm{~Hz}$. The examined solution parameters are the coefficients of lift, $\mathrm{C}_{\mathrm{L}}$, and moment coefficients, $\mathrm{C}_{\mathrm{m}}$. Table 2 shows the mean values for the oscillating lift and moment coefficients obtained from the test cases. The number of mesh elements selected for this simulation study is 200000 .

Table 2 Mesh Convergence Result.

\begin{tabular}{|c|c|c|}
\hline Num. of elements & $\mathrm{C}_{\mathrm{L}}$ & $\mathrm{C}_{\mathrm{m}}$ \\
\hline 50,000 & 0.30283 & 0.017321 \\
\hline 100,000 & 0.30633 & 0.017427 \\
\hline 150,000 & 0.30832 & 0.017575 \\
\hline 200,000 & 0.30866 & 0.017581 \\
\hline 250,000 & 0.30891 & 0.017571 \\
\hline
\end{tabular}

In order to validate the coupling between the fluid flow and structural dynamic of the rigid airfoil, one existing stall flutter experiment performed by Poirel et al. (2008) was chosen as a validation test case. The comparison for the validation test cases is shown in Fig. 3. The simulation parameters are similar to the parameters mentioned in section 2.5. The results show a good agreement between the experimental and numerical simulation work. The comparison is reasonably good as the trend from this simulation results trail the trends observed in the experiment. The quantitative behaviour of small amplitude flutter is known to be especially sensitive to airfoil geometry, reduced frequency, maximum incidence, and airspeed. The qualitative behaviour is closely related to the boundary layer separation characteristics such as trailing edge separation.

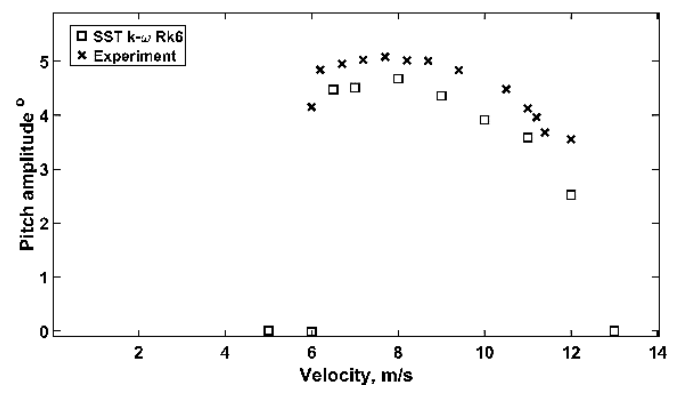

Fig. 3. LCO pitch amplitude comparison between the experiment and the RANS simulations.
There is a slight difference at the point at which the LCO manifested. Experimental observation shows LCO starts at airspeed of $6.0 \mathrm{~m} / \mathrm{s}$ while the simulation shows LCO to manifest at a slightly higher airspeed of $6.5 \mathrm{~m} / \mathrm{s}$. Minor dissimilarities between the numerical and experimental values could be caused by the $2 \mathrm{D}$ nature of the simulation that affects separation characteristics. These results are acceptable as the difference of the LCO onset speed between the experimental and numerical observations is low. Both results agreed that above $12 \mathrm{~m} / \mathrm{s}$ airspeed, no LCO is observed, and noticed no oscillations appeared at an airspeed of $13 \mathrm{~m} / \mathrm{s}$ in the simulation. Observations from Fig. 3 shows the maximum LCO amplitude is at $7.8 \mathrm{~m} / \mathrm{s}$ and it starts to decrease as the velocity increases.

\section{RESUlts}

In this section, the results of the simulation work performed on a single degree of freedom aeroelastic system are presented and discussed. First, the description and discussion revolve around the dynamic characteristic of the system where the responses, frequency contents, and bifurcation behaviour are analysed. Subsequently, the analysis and discussion of the unsteady aerodynamics characteristics such as the pitch moment coefficient, flow field and wall shear stress around the NACA 0012 undergoing oscillations are performed to better explain the aerodynamics mechanism of the stall flutter.

\subsection{Dynamic Characteristics of Responses and LCOs}

The numerical simulations in this work were simulated within the range of airspeeds between 5 $\mathrm{m} / \mathrm{s}$ and $13 \mathrm{~m} / \mathrm{s}$ and at different elastic axis positions. Within these parameters, two types of responses were observed. The first type of response shown in Fig. 4 is a decaying response. Figure 4 shows the time response for the $18 \%$ case simulated at an airspeed of $6 \mathrm{~m} / \mathrm{s}$. The response shows the system amplitude after it was released from its initial condition of $0.2^{\circ}$.

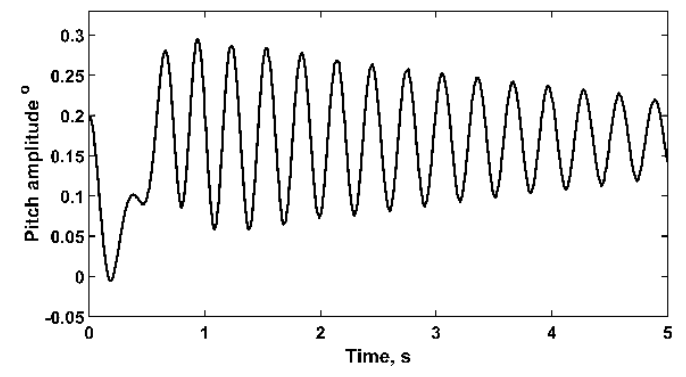

Fig. 4. Decay response at airspeed of $6 \mathrm{~m} / \mathrm{s}$ for $18 \%$ elastic axis position

The trend of the pitch amplitude of the resulting oscillation can be seen to decrease with time. Even though the amplitude did not decrease to its equilibrium point within the simulated time frame, 
the trend leads to the conclusion that the motion will eventually stop. This assumption is valid since the trend conforms to the dynamic behaviour of the aeroelastic system below the critical airspeed (Razak et al. 2011). At this airspeed, it can be said that the damping effect is not being fully negated by the energy being absorbed from the airflow. It only prolonged the oscillation. This response can be observed when the system is said to approach the onset flutter airspeed. If the simulation were to be conducted at a slightly lower airspeed, a quicker rate of amplitude decay should be observed. In this work, decaying response oscillations are plotted as zero amplitude in the bifurcation plot.

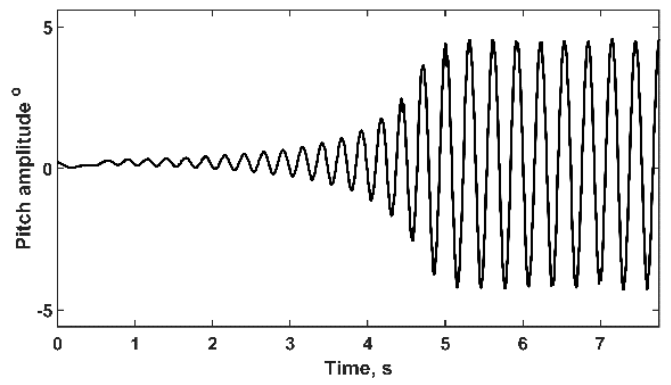

Fig. 5. LCO response at airspeed of $7 \mathrm{~m} / \mathrm{s}$ for $18 \%$ elastic axis position.

The second type of response observed was the selfsustained limit cycle oscillation. The general behaviour of this oscillation is that, after being released from its initial condition, the oscillation amplitude increases until it settles at some value as shown in Fig 5. This type of oscillation can be observed when the damping of the system has been overcome by extracting energy from the airflow. This led to the system achieving self-sustained oscillation. Self-sustained oscillation is only possible when there is more energy being absorbed into the system than the energy lost (Zorkipli et al. 2017). In this case, more energy being absorbed from the airflow than the energy being lost from the system through the effect of damping. The extra energy gained was being used to oscillate the aeroelastic system. It is also observed from Fig. 5. that the oscillation is limited in terms of its amplitude. The limitation of the amplitude or LCO is due to the existence of aerodynamic nonlinearity usually associated with the flow separation and reattachment (Dimitriadis and $\mathrm{Li}$ 2009). LCOs responses are represented by its mean oscillation amplitude in the bifurcation plot.

Figure 6 shows the bifurcation plot for the three different elastic axis positions simulated at various airspeeds. The y axis represents the oscillation amplitude while the $\mathrm{x}$-axis represents the airspeeds. The simulations revealed that all three elastic axis positions lead to self-sustain oscillation with different onset airspeeds, amplitudes and LCO termination airspeeds.

It is also noticeable from Fig. 6. the two ranges of amplitudes for the cases simulated.

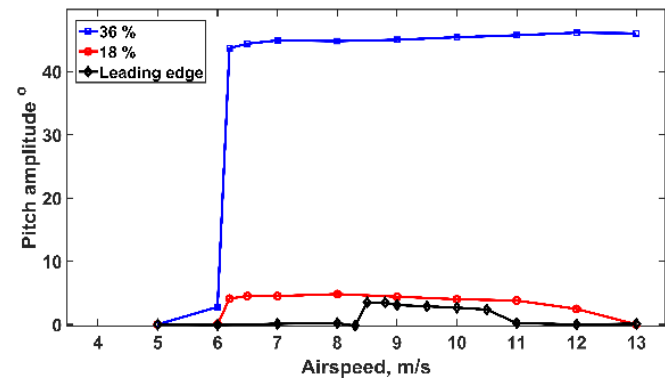

Fig. 6. Bifurcation plot for elastic axis position at the leading-edge, $18 \%$ and $36 \%$.

The range of amplitudes is classified as large and small oscillation amplitudes. As the elastic axis is shifted downstream from the leading-edge. The overall trend for the LCO amplitudes increases. A dramatic increase is evidenced when it jumps from around $5^{\circ}$ (for the $18 \%$ case) to around $43^{\circ}$ (for the $36 \%$ case). The mechanism for large oscillation amplitude is addressed in section 3.2. The jump in the amplitudes between the elastic axis at the leading-edge and at $18 \%$ of chord is attributed to the increase in the distance between the centre of pressure and the elastic axis. There are small differences in terms of LCO amplitude values between the leading-edge case and the $18 \%$ case. LCOs amplitudes in both cases are considered as small amplitude and share the same dynamic behaviour. The resulting mechanism responsible for small amplitude LCOs could originate from the separation of the boundary layer which induced the oscillations. The $36 \%$ case is classified as the largeamplitude oscillation as the pitch oscillation amplitudes reached more than $40^{\circ}$. The initial belief for this case is the mechanism that led to high amplitude oscillation is different than in previous cases.

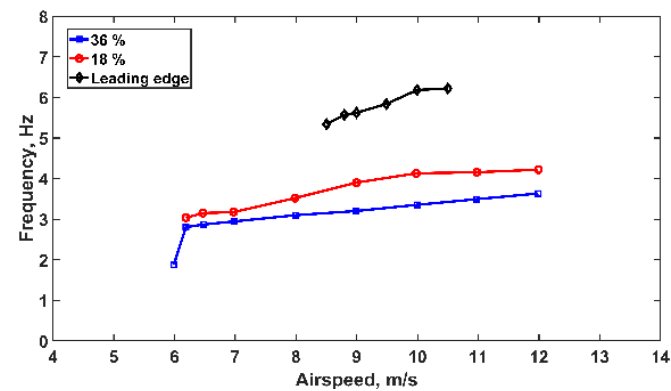

Fig. 7. LCO frequency content for elastic axis position at the leading-edge, $18 \%$ and $36 \%$ cases.

Figure 7 shows the frequency contents of the responses plotted for all elastic axis positions. The frequency content is obtained by performing system identification using the Fast Fourier Transform algorithm. The peak frequency for each LCOs response is selected and plotted against the airspeeds. The general trend shows the LCOs frequencies increases as the elastic axis shifts forward. Elastic axis position at the leading-edge resulted in the highest frequency value followed by $18 \%$ and $36 \%$ cases respectively. It can be said that changes in the 
elastic axis position affect the frequency of the stall flutter oscillation frequency.

\subsection{Dynamics of High Amplitude LCOs}

High amplitude LCOs were observed in the $36 \%$ case. At $5 \mathrm{~m} / \mathrm{s}$, the system exhibited a decay response. Two types of responses were observed which were decay responses and LCOs responses. Before large-amplitude LCOs were observed, the system started with low-amplitude LCO at $6 \mathrm{~m} / \mathrm{s}$. Figure 8 shows the response for $36 \%$ case obtained at an airspeed of $8 \mathrm{~m} / \mathrm{s}$.

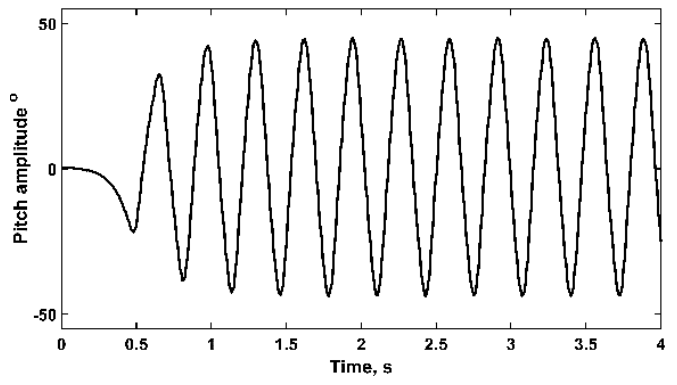

Fig. 8. LCO Response for the elastic axis $36 \%$ position.

It was observed that the amplitude of the oscillation reached around $\pm 42^{\circ}$. This high amplitude oscillation indicated that the mechanism responsible for this type of LCOs is different from the low-amplitude LCOs since all the cases were triggered with the same initial condition. Boundary layer separation could not have resulted in large-amplitude LCOs. In order to achieve that level of amplitude, the flow separation mechanism responsible could involve the manifestation of the dynamic stall phenomenon. Dynamic stall phenomenon is a phenomenon where the formation, convection and shedding of the leading-edge vortex is capable in generating a high magnitude of the moment coefficient which would increase the pitch amplitude higher (Dimitriadis and Li 2009). Furthermore, the pitch amplitude exhibited by the system was found to be higher than the static stall angle of attack for NACA 0012. The static stall angle for the chosen airfoil is known to be around $10^{\circ}$. Therefore, large flow separation must have been occurring on the airfoil. The question of whether the airfoil experienced complete or partial flow separation will be analyzed in section 3.4.

\subsection{Aerodynamics of Small Amplitude LCOs}

Analysis of the moment coefficient, $\mathrm{C}_{M}$ during oscillation can provide information on the flow of energy into the aeroelastic system. Figure 9 shows the aerodynamic moment coefficient as a function of the pitch angle at an airspeed of $7 \mathrm{~m} / \mathrm{s}$ for the $18 \%$ case. The plot shows the moment coefficient turns clockwise. This signifies that the energy is transferred from the airflow to the airfoil. The flow of extra energy into the system allows the oscillations to be sustained. From an aeroelastic point of view, it also means that positive work is being done by the aerodynamic moment as the airfoil pitches up and down. The slope of the main axis of the ellipse is also a noticeable feature. It can be interpreted as an equivalent linear static aerodynamic moment coefficient (Peristy 2014). A negative slope of the main axis of the ellipse means that the aerodynamic stiffness is positive and has a stabilizing effect.

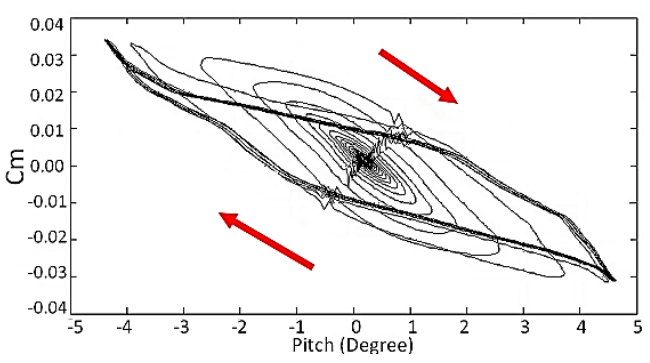

Fig. 9. $C_{M}$ versus pitch angle at $V_{\infty}=7 \mathrm{~m} / \mathrm{s}$ for $18 \%$ elastic axis position.

LCOs observed in this work are driven by nonlinearity in the aerodynamic forces since there is no nonlinearity prescribed in the structure. The source of aerodynamic nonlinearity is from the periodic separation of the flow around the airfoil. In this work, flow separation is taken as the point where the wall shear stress $\left(\tau_{w}\right)$ passed through zero and remains in the negative region. The reattachment flow is defined when the $\tau_{w}$ becomes positive from the negative region and stay positive. The exact position of flow separation was obtained from the $\tau_{w}$ at the point where it vanishes. Figure 10 shows the snapshots of the instantaneous $\tau_{\mathrm{w}}$, unsteady pressure coefficient $\left(C_{p}\right)$ and turbulent viscosity contour at 8 $\mathrm{m} / \mathrm{s}$ for the $18 \%$ case. The instantaneous pitch angle selected are $-0.4^{\circ}, 3.5^{\circ}$ and $4^{\circ}$. The arrows represent the pitching direction of the airfoil when the snapshots are taken. $C_{p}$ and $\tau_{\mathrm{w}}$ are plotted against the airfoil chord length represented by $\mathrm{x} / \mathrm{c}$. The red and blue line represents the values for the upper and lower airfoil surface respectively.

The instantaneous wall shear stress plotted in Fig. 10 for $\theta=-0.4^{\circ}$ shows the laminar boundary layer separation occurred in the vicinity of $x / c \approx 0.7$ on the lower and surface as the airfoil starting to pitch down. There is no flow reattachment at the trailing edge for the upper surface, reattachment occurred at the lower surface according to the definition used here as $\tau_{w}$ crossed into the positive region when the airfoil about to pitch upward. The small hump on the instantaneous $C_{p}$ plot between $\mathrm{x} / \mathrm{c}=0.7$ and 0.8 indicates the existence of the laminar separation bubble (LSB) on the lower side of the airfoil.

At $\theta=3.5$ during the airfoil pitching up, flow separation is observed to begin at $\mathrm{x} / \mathrm{c} \approx 0.45$ on the upper surface and no flow reattachment is observed as the $\tau_{w}$ stays in the negative region. The hump for the upper surface plot indicates the presence of turbulent flow and the transition process from laminar to turbulence occurring near the trailing edge. The flow on the opposite side of the airfoil is 

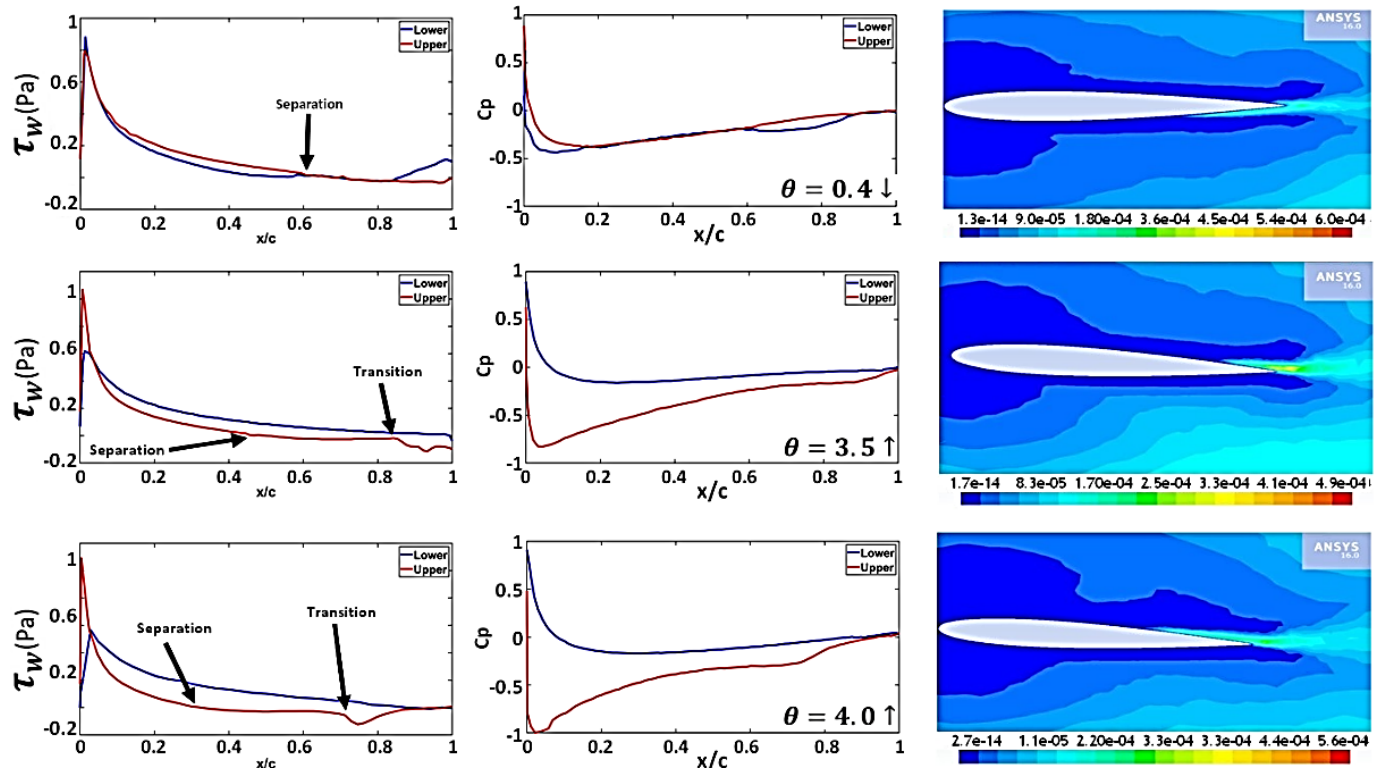

Fig. 10. Wall shear stress plot (left), Pressure coefficient (center) and turbulent viscosity contour (right) snapshots for the $18 \%$ elastic axis position simulated at $8 \mathrm{~m} / \mathrm{s}$.
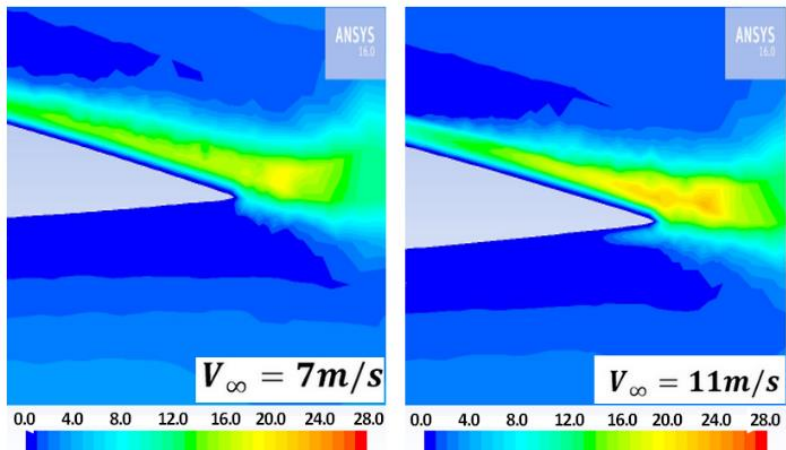

Fig. 11. Turbulent viscosity ratio at $7 \mathrm{~m} / \mathrm{s}$ (left) and $11 \mathrm{~m} / \mathrm{s}$ (right).

fully attached without any separation along the airfoil.

At $\theta=4.0$, similar behaviour is observed. Flow separation on the upper surface happened earlier at $\mathrm{x} / \mathrm{c} \approx 0.3$ and the transition to turbulence also happened earlier, increasing the pitching moment in the process. A higher pressure difference is observed along the length of the airfoil in this snapshot causing the airfoil to reach its maximum pitch angle. The turbulent viscosity contour plotted near the trailing edge for the three snapshots show higher values hinting towards the nature of the flow transiting from laminar to turbulent. Figure 11 shows the comparison of turbulent viscosity ratio contour when the airfoil is pitching upward at the maximum pitch angle at two different airspeeds. The turbulent viscosity ratio is observed to be highest at the trailing edge as the transition to turbulence only occurred at the near wake region near the trailing edge. As the airspeed increases, the overall Reynolds number also increases. This trend is mirrored in the turbulent viscosity ratio trend. This observation was also made by Poirel and Yuan (2010).
Airspeed of $11 \mathrm{~m} / \mathrm{s}$ recorded the highest viscosity ratio of 25 compared to the airspeed of $7 \mathrm{~m} / \mathrm{s}$ case which only recorded 16.5. As mentioned earlier, the

amplitude decreases as the airspeed increases. A possible explanation for this phenomenon is the increase of the turbulence level at the near wake of the airfoil. At higher Reynolds number airspeed, the separation point is clearly defined due to the increased turbulent viscosity ratio which tends to push the point where the laminar separation point occurring further back. At velocity above $12 \mathrm{~m} / \mathrm{s}$, the oscillation disappeared caused by the increasing turbulence level in the near wake regime of the airfoil where the laminar separation point has reached the trailing edge. One important thing to note during the low amplitude LCOs is the flow separation only occurs around the trailing edge.

\subsection{Aerodynamics of High Amplitude LCOs}

Figure 12 shows the snapshots of the instantaneous $\tau_{\mathrm{w}}$, unsteady $C_{p}$ and turbulent viscosity contour at $8 \mathrm{~m} / \mathrm{s}$ for the $36 \%$ case. The examination of the 

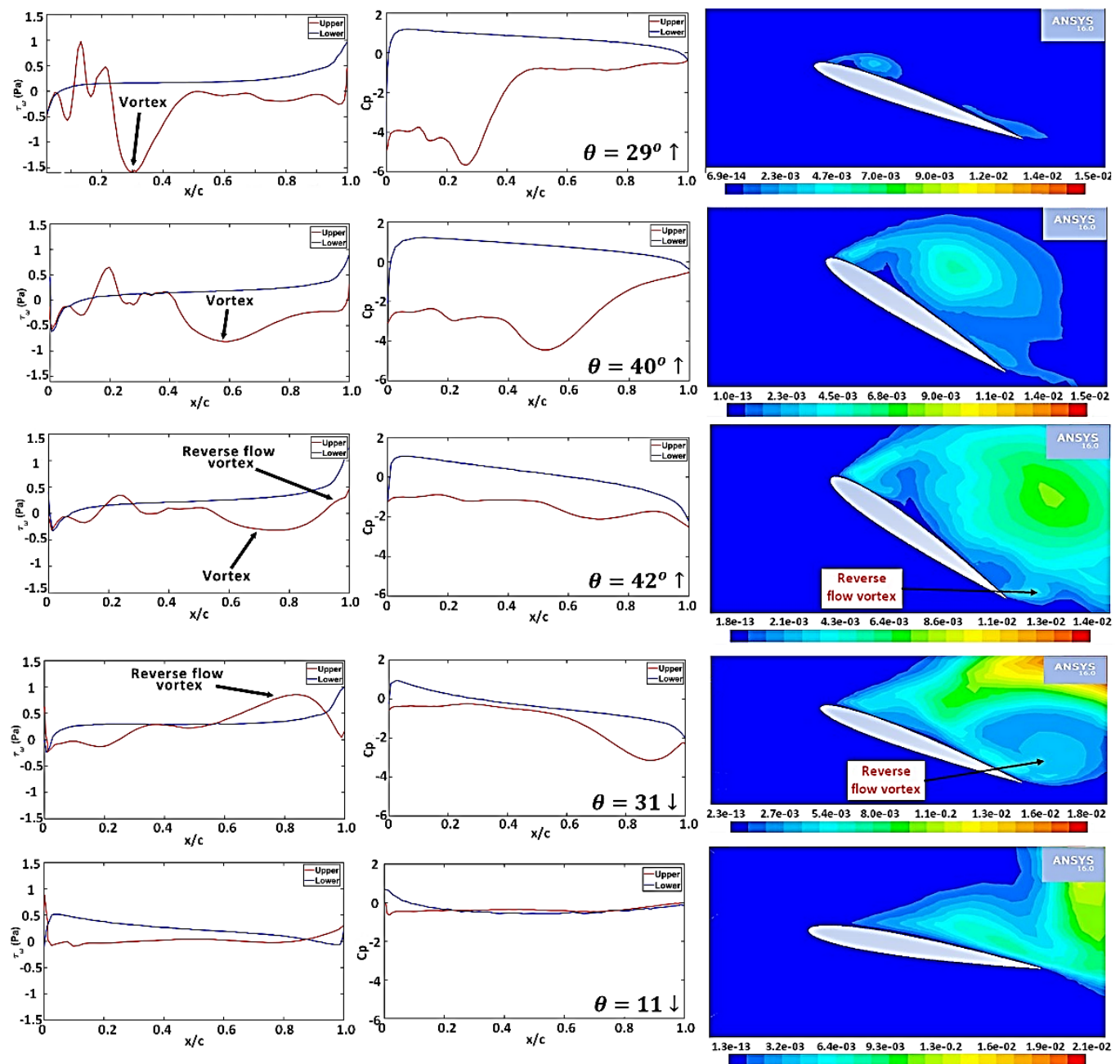

Fig. 12. Wall shear stress plot (left), pressure coefficient (center) and turbulent viscosity contour (right) snapshots for the $36 \%$ elastic axis position simulated at $8 \mathrm{~m} / \mathrm{s}$.

separation mechanism and dynamic stall phenomena occurring during high amplitude LCOs is performed by looking at the viscosity contour, wall shear stress and pressure distribution around the airfoil surface. This examination utilizes 5 instantaneous snapshots at pitch amplitudes of $29^{\circ}, 40^{\circ}, 43^{\circ}, 31^{\circ}$, and $11^{\circ}$ during upward and downward pitching as shown by the arrows.

At $29^{\circ}$, the airfoil pitch angle has exceeded its static stall angle and yet, the flow just beginning to separate. There is a small area at the trailing edge where the flow might have been separated. At the leading-edge, there is clear evidence of the formation of the leading-edge vortex. The evidence is supported by the wall shear stress plot and $C_{p}$ plot. In both plots, the location of the leading-edge vortex shows a drastic decrement in wall shear stress and sharp decreases in pressure coefficient. Flow separation is clearly indicated when the $\tau_{w}$ crosses the zero axis. In this case, the separation is in the form of the formation of leading-edge vortex. A reverse hump-like shape in the $C_{p}$ plot at $\mathrm{x} / \mathrm{c}=0.3$ shows the presence of vortex on the upper airfoil surface. The vortex is clearly captured by the viscosity contour plot when the airfoil pitch amplitude reaches $40^{\circ}$. The size of the vortex has grown and covering almost the entire airfoil upper surface. The position of the vortex core has moved from the $\mathrm{x} / \mathrm{c}=0.3$ to beyond the mid chord roughly at $\mathrm{x} / \mathrm{c}=0.55$. The big downward hump in the $C_{p}$ plot signifies a low suction region with the increase in the $C_{p}$ magnitude. The area of the hump is also larger corresponding to the size of the vortex. The maximum peak lift of the entire airfoil is achieved around this instantaneous position. This stage is known as the vortex convection on the airfoil surface.

The shedding stage of the leading-edge vortex is visible in the $42^{\circ}$ snapshot shown in Fig. 12. The vortex has moved further away from the airfoil surface. The size of the vortex appears to increase to be larger than the previous snapshot. Instead of leaving the airfoil via the trailing edge of the airfoil, the vortex, in this case, detaches around $0.75 \mathrm{c}$ due to the combination of high pitch angle and local flow direction. The detachment of the leading-edge vortex also means the starts of the flow separation. This is evidenced by the small reverse flow appearing at the trailing edge of the airfoil. As the vortex position was further away from the airfoil, its effects also started 
to diminish. This is indicated in the pressure distribution plot. The wall shear stress values around the leading-edge region show that the flow has separated. As noticed at $\mathrm{x} / \mathrm{c}=0.95$, the presence of a small reversal flow vortex starts to develop at the trailing edge. It indicates that the airfoil about to pitch down and a sudden decrease in the lift coefficient occurs at a maximum angle of attack. The vortex is eventually shed downstream causing a sudden loss of vertical force and followed by flow reversal. The formation of a reversal flow vortex at the trailing edge causes the drop in the lift and results in the airfoil starting to pitch downward.

Snapshot taken at $31^{\circ}$ shows the vortex has been detached from the airfoil and become entrained into the turbulent wake downstream of the airfoil. The flow on the upper surface experiences flows separation. The separation causes a sudden loss of lift, a peak in the pressure distribution and a maximum in nose-down pitching moment. It also induces a reversal flow vortex at the trailing edge. This reverse flow vortex originates from the flow interaction between the low-pressure region on the upper surface and the high-pressure region on the lower surface. This vortex then grew larger as indicated by the $\tau_{w}$ plot at $\mathrm{x} / \mathrm{c}=0.83$. Reversal flow near the trailing edge has been found to contribute to the recovery of lift but only slightly (Thomareis and Papadakis 2017). The level of pressure observed at $\mathrm{x} / \mathrm{c}=0.85$ also helps to pitch the airfoil downward.

As the airfoil pitched further downward, the flow at the trailing edge can be seen to begin the recovery process as shown in the snapshot of $11^{\circ}$. The attachment starts from the trailing edge. This led to the start of the recovery process for pressure distribution along the airfoil upper surface as shown in the $C_{p}$ plot. It shows that pressure recovery is not fully achieved since the lift coefficient crossed the zero line of the y axis shortly after and the cycle repeats itself for the lower surface of the airfoil. The dynamic stall phenomena observed occurs twice for each oscillation cycle for the upper and lower surface of the airfoil.

Studies have shown that the deep dynamic stall phenomenon as coined by McCroskey (1981) is characterized by the shedding and convection over the upper surface of the airfoil of a vortex-like disturbance which includes a highly non-linear and fluctuating pressure field (Yarusevych et al. 2009; Schreck and Helin 1994; Razak et al. 2011). Deep dynamic stall is well defined when the reduced frequency, amplitude and maximum incidence are sufficiently high as observed in the high amplitude LCOs. The manifestation of the leading-edge vortex caused the unsteady fluctuations in the aerodynamic load to be very large leading to high amplitude oscillation. Pellegrino and Meskell (2017) The magnitude and harmonic content of the fluctuating loads depend on the angle of attack.

\section{Conclusion}

The present simulation work has demonstrated the effects of the elastic axis positions on the stall flutter phenomenon. Different elastic axis positions lead to different kinds of stall flutter dynamics. The two distinct dynamics of the stall flutter phenomenon observed occur at a similar range of airspeeds and giving rise to LCOs of different amplitudes and frequency contents.

The fundamental frequency of the LCOs is affected by the flow separation mechanism. The onset frequencies for the elastic axis located at $36 \%, 18 \%$ and leading edge are $2.78 \mathrm{~Hz}, 2.94 \mathrm{~Hz}$ and $5.41 \mathrm{~Hz}$. The frequency is not exclusively determined by the pitch natural frequency at the zero airspeed. The frequencies increase as the airspeed increases. The onset airspeed also differs when the elastic axis position changes such that the $36 \%$ and $18 \%$ cases onset are at $6.2 \mathrm{~m} / \mathrm{s}$ while the leading edge onset is at $8.2 \mathrm{~m} / \mathrm{s}$. The bifurcation plot does indicate a weak onset airspeed dependency on the elastic axis position. This could be due to the nonlinear effects governing the phenomenon. Further work is required in order to establish a dependency of stall flutter onset airspeed to the elastic axis position.

The foundation of the manifestation of stall flutter is the mutual interaction of elastic structures with nonlinear aerodynamics. In this study, the source of aerodynamic nonlinearity has been shown to originate from the flow separation. Two types of flow separation have been observed, primarily the boundary layer separation and the dynamic stall phenomenon. Boundary layer separation is responsible for the low amplitude LCOs where the amplitudes only reach around $4.0^{\circ}$. It only occurs around the airfoil trailing edge although the pitch amplitudes are still lower than the NACA 0012 static stall angle. Dynamic stall phenomenon that leads to high amplitude LCOs around $45.0^{\circ}$ causes the flow to separate over the entire airfoil surface. The formation of the leading-edge vortex strongly amplifies the lift generated by the airfoil leading to high oscillation amplitudes.

\section{ACKNOWLEDGEMENTS}

This work was supported by the Bridging Grant No. 6316310 provided by University Sains Malaysia.

\section{REFERENCES}

Abu Bakar, H., A. Abas and N. A. Razak (2019) Effectiveness of stent in the treatment of renal artery aneurysm using FSI simulation. Journal of Applied Fluid Mechanics 12(4), 1347-1358.

Aftab, S. M. A., A. S. Mohd Rafie, N. A. Razak and K. A. Ahmad (2016). Turbulence Model Selection for Low Reynolds Number Flows. PLOS ONE 0153755.

Ahmad, K. A., W. McEwan, J. K. Watterson and J. Cole (2005). RANS turbulence models for pitching airfoil. WIT Transactions on The Built Environment 84.

ANSYS Inc. (2015). ANSYS Fluent Theory Guide. ANSYS Document 15,80 . 
M. K. H. M. Zorkipli et al. / JAFM, Vol. 14, No. 6, pp. 1679-1689, 2021.

ANSYS Inc. (2010). Introduction to ANSYS FLUENT Turbulence Modeling. 13, 1-22.

Canonsburg, A. D. (2015). ANSYS Fluent Customization Manual 724-746.

Chopra, A. K. (1996). Dynamics of structures: theory and applications to earthquake engineering. Prentice Hall, Des Moines, U.S.A.

Dimitriadis, G. and J. Li (2009). Bifurcation behavior of airfoil undergoing stall flutter oscillations in low-speed wind tunnel. AIAA Journal 47(11), 2577-2596.

Ericsson, L. E. and J. P. Reding (1980). Dynamic stall at high frequency and large amplitude. Journal of Aircraft 7(3), 136-142.

Gang, C. Z., W. Lin-kai and X. F. Long (2019). Fluid-Structure interaction numerical simulation of bridge wind-induced vibration based on CV Newmark- $\beta$ method. Journal of Highway and Transportation Research and Development 13(2).

Kinsey, T. and G. Dumas (2008). Parametric study of an oscillating airfoil in a power-extraction regime. AIAA Journal 46(6), 1318-1330.

Mccroskey, W., K. Mcalister, L. W. Carr, S. L. Pucci, O. Lambert and R. F. Indergrand (1981). Dynamic stall on advanced airfoil sections. Journal of The American Helicopter Society 26, 40-50.

McCroskey, W. J. (1981). The phenomenon of dynamic stall. NASA Technical Memorandum 81264.

Menter, F. R. (1994). Two-equation eddy-viscosity turbulence models for engineering applications. AIAA Journal 32(8), 1598-1605.

Newmark, N. M. (1959). A Method of Computation for Structural Dynamics. ASCE Journal of the Engineering Mechanics Division 85(3), 67-94.

Pellegrino, A. and C. Meskell (2013). Vortex shedding for a pitching wind turbine blade section at high angles of attack. Journal of Wind Engineering and Industrial Aerodynamics 121, 131-137.

Peristy, L. H. (2014). Examination of Small and
Large Amplitude Aeroelastic Oscillations in Pitch of A NACA0012 Airfoil. M.Sc. Thesis, Royal Military College, Canada.

Poirel, D., Y. Harris and A. Benaissa (2008). Selfsustained aeroelastic oscillations of a NACA0012 airfoil at low to moderate Reynolds numbers. Journal of Fluids and Structures 24(5), 700-719.

Poirel, D. and W. Yuan (2010). Aerodynamics of laminar separation flutter at a transitional Reynolds number. Journal of Fluids and Structures 26(7-8), 1174-1194.

Raggett, J. D. (1973). Linear theory of stall flutter. AIAA Journal 11(5), 733-735

Razak, N. A., T. Andrianne and G. Dimitriadis (2011). Flutter and stall flutter of a rectangular wing in a wind tunnel. AIAA Journal 49(10), 2258-2271.

Razak, N. A. and G. Dimitriadis (2013). Aeroelastic response of a 2-DOF wing with structural and aerodynamic nonlinearity. In Proceedings of International Forum on Aeroelasticity and Structural Dynamics, Bristol, U.K. 1-13.

Schreck, S. J. and H. E. Helin (1994). Unsteady vortex dynamics and surface pressure topologies on a finite pitching wing. Journal of Aircraft 31(4), 899-907.

Thomareis N. and G. Papadakis (2017). Effect of trailing edge shape on the separated flow characteristics around an airfoil at low Reynolds number: A numerical study. Physics of Fluids 29(1), 014101.

Yarusevych, S., P. E Sullivan and J. G. Kawall (2009). On Vortex shedding from an airfoil in Low-Reynolds-Number flows. Journal of Fluid Mechanics 632, 245-271.

Zorkipli, M. K. H. M., A. F. Razaami and N. A. Razak (2017). Experimental and numerical simulations of NACA 0018 airfoil undergoing stall flutter with structural nonlinearity. In Proceedings of International Forum on Aeroelasticity and Structural Dynamics, Como, Italy. 